\title{
Distorted superconducting nodal line on a single Fermi surface in the anisotropic organic superconductor $\lambda$-(BETS $)_{2} \mathbf{G a C l}_{4}$
}

\author{
Dita Puspita Sari $\odot,{ }^{1,2, *}$ Retno Asih $\odot,{ }_{, 2, \dagger}^{1,2}$ Ko-ichi Hiraki, ${ }^{3}$ Takehito Nakano $\odot,{ }^{4}$ Yasuo Nozue $\odot,{ }^{2}$ \\ Yasuyuki Ishii $\odot,{ }^{5}$ Adrian D. Hillier $\odot,{ }^{6}$ and Isao Watanabe ${ }^{1,2}$ \\ ${ }^{1}$ Meson Science Laboratory, RIKEN Nishina Center, 2-1 Hirosawa, Wako, Saitama 351-0198, Japan \\ ${ }^{2}$ Department of Physics, Osaka University, Osaka 560-0043, Japan \\ ${ }^{3}$ Department of Physics, Fukushima Medical University, Fukushima 960-1295, Japan \\ ${ }^{4}$ Graduate School of Science and Engineering, Ibaraki University, Mito 310-8512, Japan \\ ${ }^{5}$ College of Enginnering, Shibaura Institute of Technology, Saitama 337-8570, Japan \\ ${ }^{6}$ ISIS Facility, Rutherford Appleton Laboratory, Chilton, Didcot, Oxon, OX11 0QX, United Kingdom
}

(Received 12 September 2020; revised 5 November 2021; accepted 12 November 2021; published 17 December 2021)

\begin{abstract}
The superconducting (SC) gap structure appearing in systems with single Fermi surface (FS) is generally described by the single gap function with $s$ - or $d$-wave symmetry. The organic superconductor $\lambda$-(BETS $)_{2} \mathrm{GaCl}_{4}$ [BETS $\left.=\left(\mathrm{CH}_{2}\right)_{2} \mathrm{~S}_{2} \mathrm{Se}_{2} \mathrm{C}_{6} \mathrm{Se}_{2} \mathrm{~S}_{2}\left(\mathrm{CH}_{2}\right)_{2}\right]$ endeavors to examine a novel SC gap structure on the distorted single cylindrical FS. Here, we show an example of the formation of the distorted SC nodal line by using the positive muon spin rotation $\left(\mu^{+} \mathrm{SR}\right)$ spectroscopy on $\lambda$-(BETS $)_{2} \mathrm{GaCl}_{4}$. Our analysis method of the $\mu^{+} \mathrm{SR}$ data reveals that the nodal line has a narrower width than that of the traditional $d$-wave by the steepness factor of 4.6(2.1), and a flat part with the maximum gap exists. We found that the amplitude of the SC gap is $2 \Delta / k_{\mathrm{B}} T_{c}=3.9(2)$ and the in-plane penetration depth is $\lambda_{\mathrm{ac}}(0)=560(5) \mathrm{nm}$. Our present study gives insight into the relation of the FS distortion and the unusual Cooper pair formation mediated by the anisotropic spin fluctuations.
\end{abstract}

DOI: 10.1103/PhysRevB.104.224506

\section{INTRODUCTION}

The superconducting (SC) gap state appearing in unconventional superconductors is one of the hardest issues in condensed matter physics. Since the first discovery of the $d$-wave $\mathrm{SC}$ state with nodal line in $\mathrm{CeCu}_{2} \mathrm{Si}_{2}$ [1], it has also been reported in the $\mathrm{Cu}$ - and $\mathrm{Fe}$-based [2-7] and organic $\kappa-(\mathrm{ET})_{2} \mathrm{Cu}_{2}(\mathrm{NCS})_{2}\left[\mathrm{ET}=\left(\mathrm{CH}_{2}\right)_{2} \mathrm{~S}_{2} \mathrm{~S}_{2} \mathrm{C}_{6} \mathrm{~S}_{2} \mathrm{~S}_{2}\left(\mathrm{CH}_{2}\right)_{2}\right]$ superconductors [8]. Unconventional pairing mechanisms of these superconductors can be explained by the spin fluctuations mediating the Cooper pair [6]. The criterion of the $d$-wave nodal state as a consequence of the unconventional Cooper-pairing mechanism and suppressed superfluid density has been debated in the case of Fermi surface (FS) becoming an imperfect spherical or cylindrical shape due to the lower crystal symmetry. Theoretical and experimental results suggested deviations from the traditional $d$-wave symmetry discussing an anisotropic mixed symmetry [2,3,9], additional broken symmetry [3], pseudogap [5,6], or multi-SC gaps $[4,7]$.

\footnotetext{
* Present address: College of Engineering, Shibaura Institute of Technology, Saitama 337-8570, Japan; dita@ shibaura-it.ac.jp

${ }^{\dagger}$ Present address: Department of Physics, Institut Teknologi Sepuluh Nopember, Surabaya 60111, Indonesia.
}

Published by the American Physical Society under the terms of the Creative Commons Attribution 4.0 International license. Further distribution of this work must maintain attribution to the author(s) and the published article's title, journal citation, and DOI.
Among strongly correlated systems, organic superconductors endeavor to investigate new SC gap states with lower FS symmetry [15]. Recently, interests have increased in the highanisotropic organic superconductor, $\lambda$-(BETS) ${ }_{2} \mathrm{GaCl}_{4}$ (shortly $\lambda$-Ga), the type-II superconductor with $T_{c} \sim 5.5 \mathrm{~K} \mathrm{[16].} \mathrm{Fig-}$ ure 1 shows the crystal and electronic structures of $\lambda$-Ga. The BETS molecules form the $\lambda$-type stacking in the conducting plane, each of which stacks side-by-side along the $a$ axis [16-19] shown in Figs. 1(a) and 1(b). The highest occupied molecular orbital (HOMO) is constructed by the $\pi$-orbital and $3 / 4$-filled. The dimerization allows the system to be treated as $1 / 2$-filled [10]. The electronic state of $\lambda$-Ga can be described by the Hubbard model with the strong Coulomb interaction $U$ inside the BETS dimer [17,18]. The relatively narrow bandwidth $W$ maintains near the Fermi level and partially nests FS, as plotted numerically by density functional theory calculation (DFT) in Fig. 1(c) [10]. The $U / W$ of $\lambda$-Ga is $0.89[10,17]$. Comparing with $\kappa$-(ET) $)_{2} \mathrm{Cu}_{2}(\mathrm{NCS})_{2}, \lambda$-Ga has the lower crystal symmetry and its HOMO is more widely spread within the dimer. The folded Brillouin zone (BZ) is no longer rectangular cuboid and becomes parallelepiped distorting the shape of FS to be the elliptic cylinder. The intermolecular interactions in $\lambda$-Ga described by the transfer integrals between dimers do not form an anisotropic triangular lattice [17,20-22] shown in Fig. 1(b) [10]. This causes orbital mixings within folded BZ [16-18,23]. Therefore overlapped FS is disconnected at the crossing point in the diagonal direction of $\mathrm{BZ}$ and separated into the quasi-one-dimensional (q1D) open sheet (blue lines) and q2D close pocket (black lines). This is approximately $14 \%$ of the $\mathrm{BZ}$ consistent with 

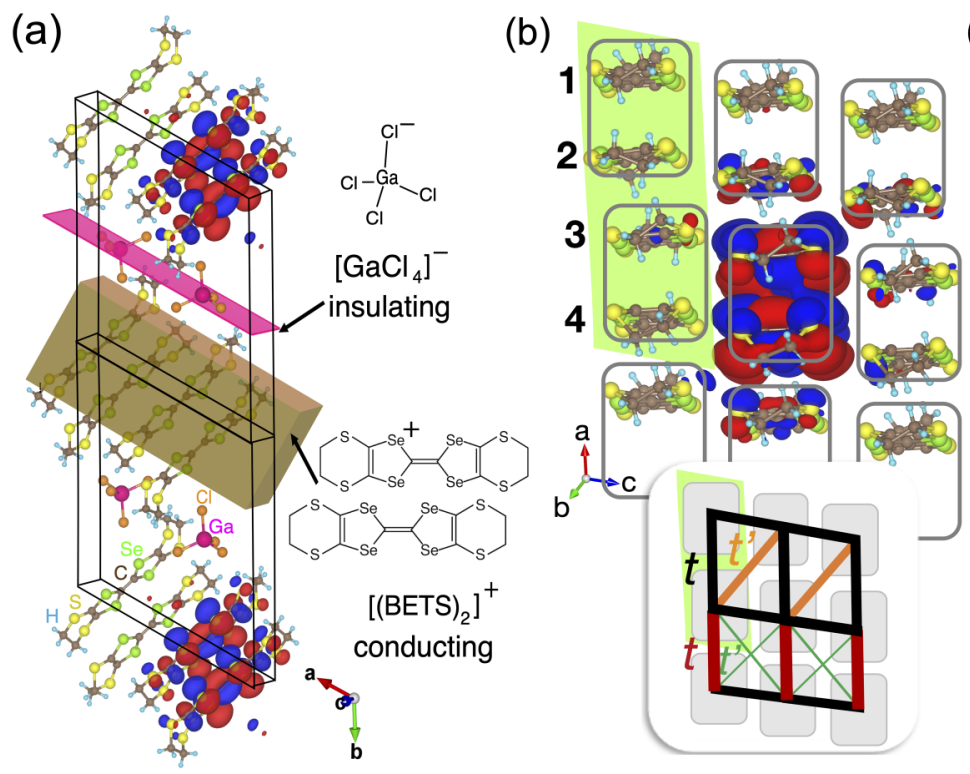

(c)

FIG. 1. (a) Crystal structure of $\lambda$-(BETS) $)_{2} \mathrm{GaCl}_{4}$. Magenta and brown layers are $\left[\mathrm{GaCl}_{4}\right]^{-}$insulating and $\left[\left(\left[\left(\mathrm{CH}_{2}\right)_{2} \mathrm{~S}_{2} \mathrm{Se}_{2} \mathrm{C}_{6} \mathrm{Se}_{2} \mathrm{~S}_{2}\left(\mathrm{CH}_{2}\right)_{2}\right]\right)_{2}\right]^{+}$conducting layers, respectively. (b) Conducting plane of $\lambda$-(BETS $)_{2} \mathrm{GaCl}_{4}$. Cyan area is the unit cell. BETS molecule 1-2 and 3-4 form a dimer. The blue and red lobes indicate the maximally localized Wannier orbital of the $\pi$ orbital on the BETS dimer (positive: blue, negative: red). In the inset, the first (second) nearest neighbors transfer integrals are indicated by black $t$ (orange $t^{\prime}$ ), with $t^{\prime} / t=0.8$. Another sublattice with the red $t$, cyan $t^{\prime}$, and $t^{\prime} / t=0.2$ alternates in the $a$ direction. (c) The 3D Fermi surface estimated from the DFT calculation [10-14].

the Subnikov-de-Hass experiment [24]. The deformation of FS was observed in the ultracold erbium atoms caused by the anisotropic dipole-dipole interaction [25]. That demonstrated anisotropic many-body phenomena possibly realizing novel topological phases or anisotropic Fermi liquid properties $[25,26]$. In $\lambda$-Ga the distorted FS structure unlocks the formation of a novel SC gap structure within a single FS.

The SC state of $\lambda$-Ga is attributed to that of $\lambda$ (BETS) ${ }_{2} \mathrm{FeCl}_{4}$ in the magnetic field above $17 \mathrm{~T}$. While $\lambda$-(BETS) ${ }_{2} \mathrm{FeCl}_{4}$ has the ground state of the antiferromagnetically long-range ordered (AFLRO) state coinciding with the metal-insulator transition at $8.3 \mathrm{~K}$ under zero field [16-18,23]. The microwave conductivity, flux-flow resistivity, heat capacity, and ${ }^{13} \mathrm{C}$-NMR studies of $\lambda$-Ga contradicted the SC gap symmetry whether it be the $s$-wave, $d$-wave, or mixed symmetry [27-31]. The ${ }^{13} \mathrm{C}$-NMR result suggested the development of the spin fluctuations in the metallic state showing the appearance of an unconventional SC state [31,32]. The SC gap symmetry is accumulated in the temperature dependence of superfluid density directly related to the inverse square of London penetration depth, $\lambda^{-2}(T)$. The $\mu^{+} \mathrm{SR}$ is a powerful tool to measure $\lambda^{-2}(T)$ in the vortex state. Here, we show the detailed analysis results of the $\lambda^{-2}(T)$ determined from $\mu^{+}$SR measurements. By using our suggested analysis method, we clarify that $\lambda$-Ga has the distorted nodal line in the SC gap structure by having a steeper nodal line than that of the traditional $d$-wave alternating with a flat part where the SC gap is maximum.

\section{EXPERIMENTAL DETAILS}

About $120 \mathrm{mg}$ single crystals synthesized by common method $[16,20]$ were packed with random orientation by a high-purity silver foil. Transverse-field (TF) $\mu^{+}$SR measurements were performed on the ARGUS and MuSR spectrometer at the RIKEN-RAL/ISIS, Rutherford Appleton Laboratory, UK [33]. The TF was applied above $T_{c}(T=$ $10 \mathrm{~K})$ perpendicular to the initial muon-spin polarization, and the sample was then cooled down maintaining TF. The $\mu^{+}$SR spectra were measured while warming from $0.3 \mathrm{~K}$ to $10 \mathrm{~K}$. The TF dependence measurement at $0.3 \mathrm{~K}$ was done to provide the information for the vortex state. The $15 \mathrm{mT}$ is within the vortex solid state $[10,34]$, while TF- $\mu^{+}$SR under $3 \mathrm{mT}$ [10] confirmed Refs. [35,36] down to $2 \mathrm{~K}$. About $25 \mathrm{mg}$ of the same sample was measured by MPMS-XL SQUID magnetometer down to $1.8 \mathrm{~K}$.

The $\mu^{+}$site $[10,37]$ was in the center of the unit cell close to the edge of the BETS molecule [10]. $\mu^{+}$could deform the local structure around the muon site, however $\mu^{+}$saw the real vortex field since the deformation is quickly gone at small distance compared to the large vortex size of $\lambda$-Ga. Our DFT calculation including $\mu^{+}$showed that the electronic metallic state, therefore the lattice shown in Fig. 1(b), hardly changes [10].

\section{EXPERIMENTAL RESULTS}

\section{A. London penetration depth}

In this section we determine the temperature evolution of penetration depth in the vortex state from temperature dependence of TF- $\mu^{+}$SR and the absolute value of in-plane London penetration depth from the field dependence measured at $0.3 \mathrm{~K}$. Figures 2 (a) -2 (c) show TF- $\mu^{+}$SR time spectra measured at 0.3 and $10 \mathrm{~K}$ under 3,6 , and $15 \mathrm{mT}$, respectively. $\mu^{+}$SR time spectra were normalized by the amplitude of the 

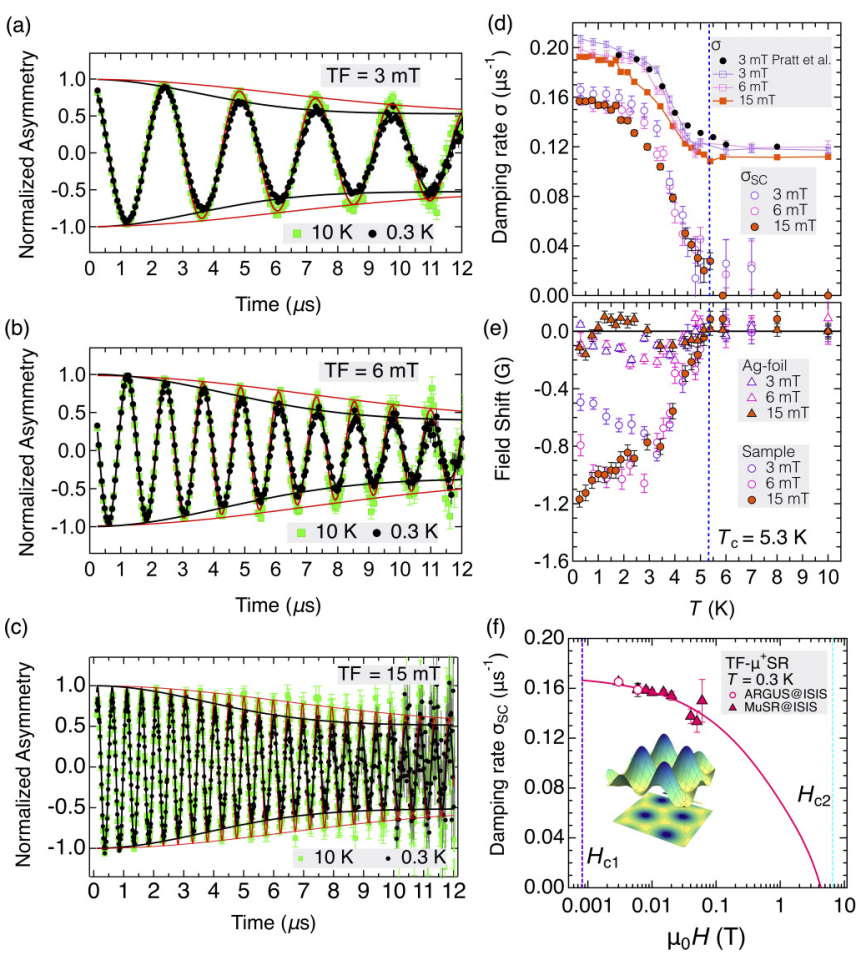

FIG. 2. (a)-(c) Normalized $\mu^{+}$SR time spectra measured in $\lambda$ (BETS) $\mathrm{GaCl}_{4}$ under the transverse field (TF) of 3, 6, and $15 \mathrm{mT}$, respectively, at $10 \mathrm{~K}$ (cyan) and $0.3 \mathrm{~K}$ (black). The red and black solid lines are the best-fitting results using the Gaussian-type-damped cosine equation. The trace of the envelope represents the damping behavior of the muon-spin precession at $10 \mathrm{~K}$ (red) and $0.3 \mathrm{~K}$ (black). (d) Temperature dependence of the damping rate, $\sigma(T)$, and that in the SC state, $\sigma_{\mathrm{SC}}(T)$, after subtracting the nuclear dipole moment part above $T_{c}$ by $\sigma^{2}=\sigma_{\mathrm{SC}}^{2}+\sigma_{\mathrm{NM}}^{2}$. The dashed line indicates $T_{c}=5.3 \mathrm{~K}$ determined from our resistivity and magnetization measurements [34]. (e) Temperature dependence of field shift extracted from the fitting parameter of $B_{1}$ and $B_{2}$ in the Eq. (1) which is shifted from those measured at $T=10 \mathrm{~K}$. (f) Transverse-field dependence of $\mu^{+}$SR damping rate in the superconducting state, $\sigma_{\mathrm{SC}}(H)$. Magenta circle and solid line are the data and fitting line by using Eq. (3) roughly estimating $H_{\mathrm{c} 2}$. Cyan and cyan broken lines are the $H_{\mathrm{c} 1}$ and $H_{\mathrm{c} 2}$ determined by the SQUID measurement in Fig. 3(a).

muon-spin precession at the determined time zero measured at $T=0.3 \mathrm{~K}$ and analyzed by applying the Gaussian-typedamped cosine equation as

$$
\begin{aligned}
A^{\mathrm{TF}}(t)= & A_{1} \exp \left[-\sigma^{2} t^{2}\right] \cos \left(\gamma_{\mu} B_{1} t+\phi\right) \\
& +A_{2} \cos \left(\gamma_{\mu} B_{2} t+\phi\right)
\end{aligned}
$$

where the first and second terms of the right side describe muon components from the sample and background, respectively. The background signal resulted from muons stopping in the high-purity silver foil surrounding the sample did not cause any damping behavior of the muon-spin precession. We obtained $A_{1}=0.48(2)$ and $A_{2}=0.51$ (3) and fixed the value for fitting time spectra at all temperatures. Here, $\gamma_{\mu} / 2 \pi=$ $135.5 \mathrm{MHz} / \mathrm{T}$ is the gyromagnetic ratio of the muon spin. $B_{1}$ and $B_{2}$ are the average magnetic field in the SC state and in the silver foil, respectively.
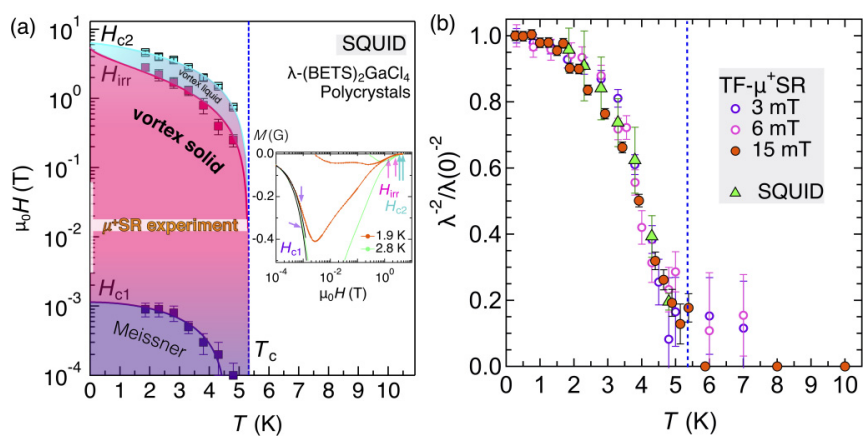

FIG. 3. (a) $H-T$ phase diagram of the superconducting state determined from magnetic susceptibility measurements. Cyan, magenta, and cyan circles show the lower, irreversible, and upper critical fields, respectively $\left(H_{\mathrm{cl}}, H_{\mathrm{irr}}, H_{\mathrm{c} 2}\right)$ determined by the isothermal field dependence of magnetization measurement ( $\mathrm{MH}$ loop) at several temperatures. Colored solid lines are the fitting lines by using Eq. (4). The inset shows two measured $M H$ loops. (b) Temperature dependence of normalized inverse square London penetration depth obtained from TF- $\mu^{+}$SR and SQUID measurement.

Figure 2(d) shows the temperature dependence of the obtained $\sigma$ from Eq. (1). The $\sigma$ is expressed as $\sigma^{2}=\sigma_{\mathrm{SC}}^{2}+\sigma_{\mathrm{NM}}^{2}$ $[5,38]$, where $\sigma_{\mathrm{SC}}$ is the damping rate in the SC state due to the inhomogeneous field distribution caused by vortices. The $\sigma_{\mathrm{NM}}$ is the damping rate caused by nuclear dipole fields distributed at the muon site [10] in the normal state $(\sigma$ at $T=10 \mathrm{~K}$ ) and was estimated to be $0.1119(12) \mu \mathrm{s}^{-1}$. The $\sigma$ at $T=8$ and $6 \mathrm{~K}$ had the same value within the error bar, shown in Fig. 2(b). The value of $0.1119 \mu \mathrm{s}^{-1}$ was applied for subtracting each data point of $\sigma$. The temperature dependence of $\sigma_{\mathrm{SC}}$ is then related to the London penetration depth $\lambda$, assuming the hexagonal vortex lattice of the quantum flux $\Phi_{0}$ [38]. In this case, we can use the Brandt equation [38,39],

$$
\sqrt{2} \sigma_{\mathrm{SC}}(T) / \gamma_{\mu}=0.06091 \Phi / \lambda^{2}(T),
$$

where $\Phi_{0}=2.068 \times 10^{-15} \mathrm{~Wb}$.

Figure 2(e) shows the temperature dependence of $B_{1}-$ $B_{1(T=10 \mathrm{~K})}$ representing the temperature dependence of the field shift. The $B_{1}$ promptly decreased below $T_{c}$ since the internal field in the superconducting state has a lower value than that of the metallic state reminiscent of the diamagnetic signal.

Figure 2(f) shows TF-dependence measurement of $\sigma_{\mathrm{SC}}(H)$ at the base temperature $0.3 \mathrm{~K}$ in the vortex region, 3-60 $\mathrm{mT}$. The $\sigma_{\mathrm{NM}}$ was subtracted from the one measured at $10 \mathrm{~K}$ for each field. From $\sigma_{\mathrm{SC}}(H)$ we can estimate the $\lambda_{\text {eff }}$ and $H_{\mathrm{c} 2}$ using the equation given by Brandt [39]

$$
\sigma_{\mathrm{SC}}(H)=4.83 \times 10^{4}\left[\left(1-\sqrt{H_{\mathrm{app}} / H_{\mathrm{c} 2}}\right)^{3}\right] \lambda_{\text {eff }}^{-2}
$$

where $\sigma_{\mathrm{SC}}$ is in $\mu \mathrm{s}^{-1}, \lambda_{\text {eff }}$ in $\mathrm{nm}$, and $H$ in Oe. The $\lambda_{\text {eff }}$ and $H_{\mathrm{c} 2}$ were estimated, respectively, to be $665 \pm 12 \mathrm{~nm}$ and $43 \pm$ $25 \mathrm{kOe}$. In order to check this estimation we estimated the critical fields in the superconducting state from magnetization measurement using SQUID.

Figure 3 summaries the $H-T$ phase diagram in $\lambda$-Ga showing $H_{\mathrm{c} 1}$, irreversibility field $H_{\mathrm{irr}}$, and $H_{\mathrm{c} 2}$ determined from the $M H$-loop measurement at several temperatures. The solid line 
is the fitting by using the two-fluid model equation,

$$
H_{i}=H_{i}(0)\left(1-\left(T / T_{c}\right)^{\mathrm{n}}\right) .
$$

The $H_{\mathrm{c} 1}, H_{\mathrm{irr}}$, and $H_{\mathrm{c} 2}$ were estimated to be $11.4 \pm 2.8 \mathrm{Oe}$, $54.8 \pm 0.1 \mathrm{kOe}$, and $63.3 \pm 0.7 \mathrm{kOe}$, respectively. The superconducting coherence length $\xi_{\text {eff }}$ is accordingly estimated from [40],

$$
H_{c 2} \approx \Phi / 2 \pi \xi_{\mathrm{eff}}^{2}
$$

yielding $\xi_{\text {eff }}=7.2(4) \mathrm{nm}$.

The $H_{\mathrm{c} 2}$ estimation from TF- $\mu^{+}$SR within the upper limit of the error bar, $H_{\mathrm{c} 2}=68 \mathrm{kOe}$, is consistent with the SQUID measurement result, $H_{\mathrm{c} 2}=64 \mathrm{kOe}$. This estimation is also consistent with the single crystal magnetoresistance measurement [41] by considering the spherical average. Information from single crystals magnetoresistance measurement [41] yields the anisotropy factor $\gamma$ of $H_{\mathrm{c} 2 \|} / H_{\mathrm{c} 2 \perp} \gtrsim 4$. It appears that the dependence on the magnetic field orientation of the $H_{\mathrm{c} 2}$ in $\lambda$-(BETS) ${ }_{2} \mathrm{GaCl}_{4}$ is qualitatively similar to the Ginzburg-Landau anisotropic effective-mass approximation [24]. When we took its spherical average in the case of random field orientation, as

$$
\frac{1}{4 \pi} \int_{0}^{2 \pi} d \phi \int_{0}^{\pi} \sin \theta d \theta \frac{H_{\mathrm{c} 2}(\theta=0)}{\sqrt{\cos ^{2}(\theta)+\gamma^{-2} \sin ^{2}(\theta)}},
$$

the $H_{\mathrm{c} 2}$ was $6.6 \mathrm{~T}$, which is consistent with the estimation from $\mathrm{TF}-\mu^{+} \mathrm{SR}$ and SQUID measurement. We determine the vortex solid and liquid region, as shown in Fig. 3(a). Accordingly, the estimation of effective London penetration depth by Eq. (3) is consistent with the prediction of the Ginzburg-Landau model, while the temperature dependence of TF- $\mu^{+} \mathrm{SR}$ was therefore done in the vortex solid region.

Furthermore, we are able to evaluate the obtained temperature dependence of TF- $\mu^{+}$SR in the field of 3,6, and $15 \mathrm{mT}$. As shown in Fig. 2(e), temperature dependence of field shift in the field of 3 and $6 \mathrm{mT}$ has some upturn at low temperature. This may be due to weak pinning effect since the system was just entering the vortex solid state. From our measurements the stable vortex solid state in $\lambda$-Ga is in the range of about $10 H_{\mathrm{c} 1}<H \ll H_{\mathrm{c} 2}$ [34]. In the field of $15 \mathrm{mT}$ the field shift curve keeps decreasing down to low temperature. The absolute value of this field shift can be estimated as $[42,43]$

$$
\Delta B_{T \rightarrow 0} \approx-0.146 \times H_{\mathrm{c} 2} \frac{1-\left(H_{\mathrm{app}} / H_{\mathrm{c} 2}\right)}{\kappa^{2}-0.069}
$$

where $\kappa=\frac{\xi}{\lambda}$ is the Ginzburg-Landau constant. In our case with $H_{\mathrm{c} 2}=63.3 \mathrm{kOe}, \lambda=665 \mathrm{~nm}$, and $\xi=7.2 \mathrm{~nm}$, the $\Delta B_{T \rightarrow 0} \approx 1.1 \mathrm{G}$, which is very consistent with the experimental result, 1.2(1) G, as shown in Fig. 2(d). It is also shown that the field shift of the $\mathrm{Ag}$ foil was almost temperature independent. Indeed, in the low- $\mathrm{T}$ region below $50 \mathrm{~K}$ such high-purity Ag foil should not show a temperature dependence.

Since we obtain the information of $\lambda_{\text {eff }}$ and $\xi_{\text {eff }}$, the $H_{\mathrm{c} 1}$ can be estimated from [40]

$$
H_{\mathrm{c} 1}=\frac{\Phi_{0}}{4 \pi \lambda_{\mathrm{eff}}^{2}} \ln \left(\frac{\lambda_{\text {eff }}}{\xi_{\text {eff }}}\right)
$$

yielding $H_{\mathrm{c} 1}=16.3 \mathrm{Oe}$. This estimation was also close to the region shown in the phase diagram of Fig. 3(a). This indicated the robustness of the $\sigma(T)$ in the TF of $15 \mathrm{mT}$, comparing with curves measured in the TF of 3 and $6 \mathrm{mT}[10,35,36]$, besides the fact that $\sigma_{\mathrm{SC}}(T F=15 \mathrm{mT}) \approx \lambda_{\text {eff }}=665 \mathrm{~nm}$.

The inset of Fig. 3(a) shows the $M H$ loop at 1.9 and $2.8 \mathrm{~K}$ in the logarithmic scale. The black solid lines are the linear fitting, and the deviations of the data from the lines indicate the $H_{\mathrm{c} 1}$. The bifurcations of the loop indicate $H_{\mathrm{irr}}$ and the vanishing of the magnetization at high field indicates the system is approaching the lower limit of the $H_{\mathrm{c} 2}$. In the vortex liquid region we deduced temperature dependence of $\lambda^{-2}$. In the vortex of a type-II superconductor with a large Ginzburg-Landau parameter the reversible magnetization for intermediate fields $H_{\mathrm{c} 1} \ll H \ll H_{\mathrm{c} 2}$ is given by London's phenomenological model [44],

$$
-4 \pi M=\frac{\Phi_{0}}{8 \pi \lambda^{2}} \ln \frac{H_{\mathrm{c} 2} \beta}{H}
$$

where the $\beta$ is a constant of order unity. Then, in the vortex liquid region, $H_{\mathrm{irr}}<H<H_{\mathrm{c} 2}$, the slope of the linear $M(\ln H)$ dependence is given by

$$
\frac{d M}{d(\ln H)}=\frac{\Phi_{0}}{32 \pi^{2} \lambda_{\mathrm{eff}}^{2}}
$$

We use this relation to analyze the logarithmic magnetization curve at various temperatures shown in the inset of Fig. 3(a). Extrapolating the data by Eq. (4) we then normalized the data by value of $\lambda(0)^{-2}$. The absolute value of $\lambda(0)^{-2}$ was smaller than one estimated from TF- $\mu^{+}$SR and the relatively large error bar was due to the sensitivity of the fitting result to the range of the linear region in the $M H$-loop data used as a fitting range. However, the overall curve of $\lambda^{-2}(T)$ is in good agreement and is plotted together in Fig. 3(b). This again puts the indication of robustness of curve shape of $\lambda^{-2}(T)$ obtained from different measurement, and therefore, the further analysis will be done for the $\lambda^{-2}(T)$ measured by the chosen $15 \mathrm{mT} \mathrm{TF}-\mu^{+} \mathrm{SR}$.

In this study, the high-anisotropic character of $\lambda$-Ga is estimated from the ratio of intra- and interlayer transfer integral [10], $t_{\|} / t_{\perp} \simeq 13 \mathrm{meV} / 0.2 \mathrm{meV} \simeq 65$, which is much higher than its in-plane anisotropy within intralayer [Fig. 1(b)], red $t /$ cyan $t^{\prime}=5$.8. Experimentally, considering large GinzburgLandau parameter, $\kappa=\lambda(0) / \xi(0) \approx 78$ [45], we refer to the $H_{\mathrm{c} 2 \|} / H_{\mathrm{c} 2 \perp} \gtrsim 4$ to determine $\lambda_{\|} / \lambda_{\perp}=4$. Therefore the $\lambda_{\text {eff }}$ can be reliably approximated as $\lambda_{\text {eff }} \simeq 1.226 \lambda_{\mathrm{ac}}$, where $\lambda_{\mathrm{ac}}$ is the London penetration depth in the conducting ac plane or $\lambda_{\perp}[5,10,46]$. From the measurement of $\sigma_{\mathrm{SC}}(H)$ we therefore obtained $\lambda_{\mathrm{ac}}=554.4 \mathrm{~nm}$.

\section{B. Superconducting gap structure}

Having the temperature dependence of the inverse square of London penetration depth, $\lambda_{\mathrm{ac}}^{-2}(T)$, we deduce in detail the accumulated information of SC gap structure in this section. The $\lambda_{\mathrm{ac}}^{-2}(T)$ is proportional to the superfluid density by the speed of light $\rho_{\mathrm{s}} c^{-2}$, and its temperature dependence of $\lambda_{\mathrm{ac}}^{-2}(T)$ contains information about the phase dependence of 

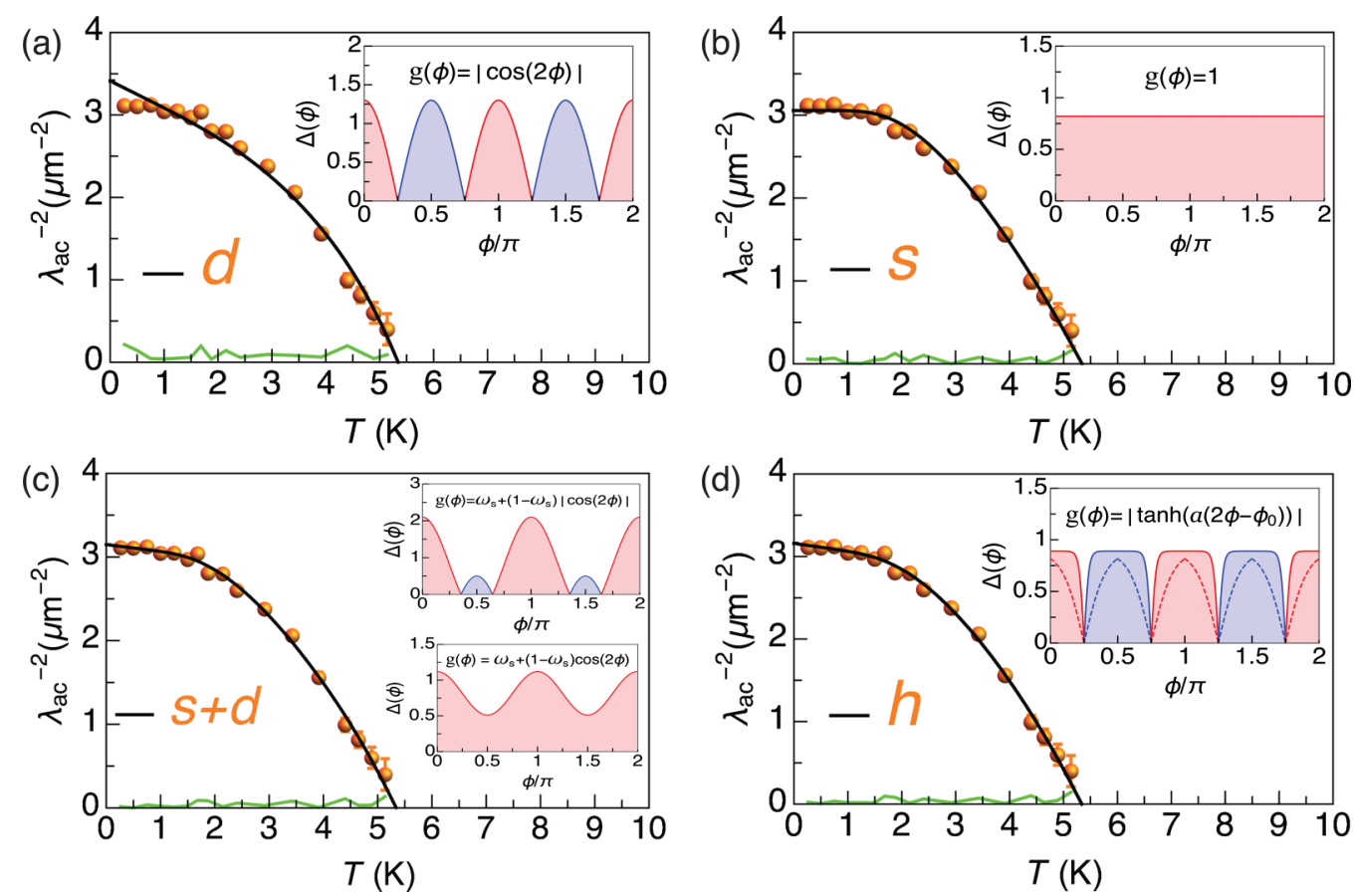

FIG. 4. Temperature dependence of the inverse square of the in-plane London penetration depth, $\lambda_{\mathrm{ac}}^{-2}=\rho_{\mathrm{s}} c^{-2}$, in $\lambda$-(BETS) $)_{2} \mathrm{GaCl}{ }_{4}$ fitted by (a) $d$-wave, (b) $s$-wave, (c) multigap $s+d$-wave, and (d) $h$-wave distorted nodal line symmetry for $a=4.6$, respectively. The lower inset in (c) shows the obtain gap structure considering the upper limit of $\sigma_{\mathrm{NM}}=0.1131 \mu \mathrm{s}^{-1}$ [10] for deducing the $\sigma_{\mathrm{SC}}$ from $\sigma$. The broken lines in (d) indicate the trajectory for $a=1$. Black solid lines are the best-fitting results for gap symmetries described by each tested gap function, $g(\phi)$. Green solid lines are the difference between data and the fit. Blue and red color areas in the insets show changes in the sign of the SC gap phase $\phi$, and the $\Delta(\phi)$ is in the unit of meV.

the SC gap by the following SC gap equation,

$$
\frac{\lambda_{\mathrm{ac}}^{-2}(T)}{\lambda_{\mathrm{ac}}^{-2}(0)}=1+\frac{1}{\pi} \int_{0}^{2 \pi} \int_{\Delta(\phi, T)}^{\infty}\left(\frac{\delta f}{\delta E}\right) \frac{E d E d \phi}{\left.\sqrt{E^{2}-\Delta_{i}(\phi, T)^{2}}\right)},
$$

where $f=\left[1+\exp \left(E / k_{\mathrm{B}} T\right)\right]^{-1}$ is the Fermi function, and $\phi$ is the angle along the Fermi surface. $\Delta_{i}(\phi, T)$ is expressed as $\Delta(T) g(\phi)$, and $i$ indicates the type of SC gap symmetry. The temperature dependence of the gap energy was set to be $\Delta(T)=\Delta(0) \tanh \left\{1.82\left[1.018\left(T_{c} / T-1\right)\right]^{0.51}\right\}$ where $\Delta(0)$ is the maximum SC gap at $T \rightarrow 0$. The only fixed parameter during the fitting process was $T_{c}$ and this was fixed to be the value determined by resistivity and susceptibility (5.3 K) [34]. The $g(\phi)$ describes the phase dependence of the gap and can be replaced by several gap functions [5,47].

Figure 4 shows the temperature dependence of $\lambda_{\mathrm{ac}}^{-2}$ fitted by several $g(\phi)$. The $\lambda_{\text {ac }}^{-2}(T)$ exhibits a rapid increase and round shape below $T_{c}$ down to approximately $2 \mathrm{~K}$ followed by a moderate increase at lower temperatures. Black solid lines are the best fitting results for gap structure described by each tested $g(\phi)$. Insets visualized the gap structure along FS, $\Delta(\phi)$. The color difference represents the sign changing in the SC phase, $\phi$.

The initial attempt to describe the temperature dependence of $\lambda_{\mathrm{ac}}^{-2}$ was done using the $d$-wave symmetry, $g(\phi)=$ $|\cos (2 \phi)|$, shown in Fig. 3(a). The fitting result showed the linear increase with lowering the temperature below $\tau_{c} / 3$. However, $\lambda_{\mathrm{ac}}^{-2}(T)$ was not as steep as that described by the $d$-wave symmetry as $T \rightarrow 0$. This lets us conclude the $d$-wave symmetry was unsuitable to describe the SC state of $\lambda$-Ga.
Instead, we tried to fit $\lambda_{\text {ac }}^{-2}(T)$ using the $s$-wave symmetry with $g(\phi)=1$. The result is shown in Fig. 3(b). The fitting line saturates with a flat ending in its temperature dependence below $T_{c} / 3$. This saturating part did not describe $\lambda_{\mathrm{ac}}^{-2}(T)$ well because it showed a slight increase as $T \rightarrow 0$. This indicated that neither the single $s$ - nor $d$-wave symmetry reliably reproduced our $\mu^{+}$SR result and that the SC gap structure of $\lambda-\mathrm{Ga}$ had an intermediate character $[34,48]$.

Accordingly, we examined the intermediate gap structure by using the two-component gap functions, $s+d$-wave. We first replaced $\Delta_{\mathrm{i}}$ in Eq. (11) by a gap function $\omega_{\mathrm{s}}+(1-$ $\left.\omega_{\mathrm{s}}\right)|\cos (2 \phi)|$. The obtained parameters were $\omega_{\mathrm{s}}=0.54, \Delta_{\mathrm{s}}=$ $0.78 \mathrm{meV}$, and $\Delta_{\mathrm{d}}=1.4 \mathrm{meV}$. However, this fitting was not statistically significant with very large $P$ value [49] above $90 \%$ related to unreasonably large standard error for each fitting parameter. Therefore, we replaced $\lambda_{\mathrm{ac}}^{-2}(T)$, which explained a multigap SC state [5,7,47], by

$$
\begin{aligned}
\lambda_{\mathrm{ac}}^{-2}(T)= & \lambda_{\mathrm{ac}}^{-2}(0)\left[\omega_{\mathrm{s}} \times \lambda_{\mathrm{ac}}^{-2}\left(T, \Delta_{\mathrm{s}}\right)\right. \\
& \left.+\left(1-\omega_{\mathrm{s}}\right) \times \lambda_{\mathrm{ac}}^{-2}\left(T, \Delta_{\mathrm{d}}\right)\right] .
\end{aligned}
$$

Ideally, when using Eq. (12) one assumes two FS and, if there is coexistence, each FS has $s$ and $d$-wave component. However, from previous test the $\omega_{\mathrm{s}}=0.54$ of the SC gap amplitude indicated that the $s$ - and $d$-wave components exist with a comparable portion. In order to minimize the degree of freedom in the fitting parameters, here, we assume $\omega_{\mathrm{s}}, \Delta_{\mathrm{s}}$ and $\Delta_{\mathrm{d}}$ are the weighting factor for the $s$-wave superconductivity, the SC gaps of $s$ - and $d$-wave SC symmetries, respectively. 
As shown in Fig. 3(c), this $s+d$-wave symmetry fitted $\lambda_{\mathrm{ac}}^{-2}(T)$ well for the whole temperature range below $T_{c}$. The obtained $\omega_{\mathrm{s}}$ was $0.71(1)$ with the $\Delta_{\mathrm{s}}=0.80(8) \mathrm{meV}$ and $2 \Delta_{\mathrm{s}} / k_{\mathrm{B}} T_{\mathrm{c}}=3.5(2)$. The $d$-wave component was $0.29(1)$ with the larger gap amplitude $\Delta_{\mathrm{d}}=1.3(5) \mathrm{meV}$ and $2 \Delta / k_{\mathrm{B}} T_{c}=$ 5.9(1.2). We note that the $\omega_{\mathrm{s}}$ here did not relate directly to the gap amplitude anymore. As we cannot distinguish whether the weighting factor could be related to some portion of FS or to the $s$-wave superconductivity, we will see next how good the Eq. (12) had been. Focusing only on the obtained gap amplitudes of $\Delta_{\mathrm{d}}$ and $\Delta_{\mathrm{s}}$, if we add those two gap symmetries the node point of $\Delta_{\mathrm{d}}$ was lifted up by $\Delta_{\mathrm{S}}$ resulting in the large (red) and small (blue) SC gap components, shown in the upper inset of Fig. 3(c).

Although this scheme could fit $\lambda_{\mathrm{ac}}^{-2}(T)$ well, considering the upper limit error of the estimated $\sigma_{\mathrm{NM}}$ the test obtained fitting parameter $\Delta_{\mathrm{s}}=0.82(4), \Delta_{\mathrm{d}}=0.30(55)$, and $\omega_{\mathrm{S}}(0)=0.94(6)$. In this analysis $\Delta_{\mathrm{S}}>\Delta_{\mathrm{d}}$ [10]. Therefore, an anisotropic gap opens all along FS without nodes, related to the minimum gap of $0.5 \mathrm{meV}$ or $5.8 \mathrm{~K}$, as shown in the lower inset of Fig. 4(c). This resulting gap structure is consistent with that using $g(\phi)=[1+b(\cos (4 \phi))]$, referred to as anisotropic-s-wave. The $b$ parameter decides how large the minimum gap opens. Our test resulted in the data can be described by $\Delta_{\mathrm{s}} 0.84 \mathrm{meV}$ and $b=0.34$, related to a minimum gap of $6.45 \mathrm{~K}$. However, the opening of the gap along FS is very hard to explain the evidence of nodes from heat capacity, ${ }^{13} \mathrm{C}-\mathrm{NMR}$, and RPA calculation [23,29-31]. The existence of nodes through the multigap $s+d$-wave model turned out to be not robust, i.e., the $s+d$-wave model could not be decisive, while the concept of single FS is the fundamental key for $\lambda$-Ga to unify experimental explanations such as the Kadowaki-Woods ratio [50]. Thus, such a multibandlike scheme [7] is unlikely for SC $\lambda$-Ga. For complete analysis considering the upper and lower limit of the error bar of $\sigma_{\mathrm{NM}}$, see Supplemental Material Fig. S4, Fig. S5, Table S2, and Table S3. [10].

In order to describe the intermediate SC state in $\lambda$ $\mathrm{Ga}$, Aizawa et al. theoretically suggested to use several well-known SC single gap functions mediated by the spin fluctuations between BETS molecules [23]. They proposed the appearance of two types of nodal line with a large component of a full-gap state. Node positions in single FS were in the diagonal direction of BZ close to $\pi / 4$, and the SC phase has only the twofold symmetry due to the distorted FS.

Consequently, we promote the single component hyperbolic tangent function function, $g(\phi)=\left|\tanh \left(a\left(2 \phi-\phi_{0}\right)\right)\right|$, to analyze $\lambda_{\mathrm{ac}}^{-2}(T)$. We sectionalized the range of integration in the SC gap equation. The phase shift $\phi_{0}$ was fixed to be $\pi / 2$ for the first integration range, from 0 to $\pi / 2$. The $\phi_{0}$ shifts by $\pi$ for every next integration range. Because a hyperbolic function is used, we simply named this distorted nodal line symmetry the " $h$-wave." It has intersecting and constant parts in relation to $\phi$. The former and latter describe the nodal line and flat part of the SC gap, respectively. Here $a$ is the factor describing the steepness of the nodal line compared with that of the $d$-wave symmetry. In the case of $a=1$, the $h$-wave symmetry produces the same nodes width as of using $g(\phi)=|\cos (2 \phi)|$. The $h$-wave fitting result excellently described the $\lambda_{\mathrm{ac}}^{-2}$ with the steepness factor $a=4.6(2.1)$, shown in Fig. 4(d). For visualization, the broken line in the inset of Fig. 4(d) plotted the case of $a=1$ with gap amplitude $\Delta_{h}(0)$. It becomes clear that the $h$-wave node is steeper than that of the $d$-wave node but having a flat part where the gap is maximum. The steep nodal line and flat structure well describe the moderate increase below $2 \mathrm{~K}$ and round shape starting just below $T_{c}$, respectively, in the curve of $\lambda_{\mathrm{ac}}^{-2}(T)$. Among others, the $h$-wave model obtained the best reduced $\chi^{2}$, defined as $\chi^{2} /(v-u)$ where $v$ is the number of data points and $u$ is the fitting-parameter degree of freedom. The reduced $\chi^{2}$ approaches unity as the model describes well the data [49].

Furthermore, although the obtained fitting parameter of the $h$-wave model, $a=4.6(2.1)$, has a relative error bar about $50 \%$, and within two standard deviations it is compatible with $a=1$, from the fitting we also obtained the $h$-wave SC gap amplitude $\Delta_{\mathrm{h}}(0)=0.89(4) \mathrm{meV}$ and $\lambda_{\mathrm{ac}}^{-2}(0)=$ $3.16(5) \mu \mathrm{m}^{-2}$. If we use $a=1$ or $a=2.15$ with $\Delta_{\mathrm{h}}(0)=$ $0.89(4) \mathrm{meV}$ and $\lambda_{\mathrm{ac}}^{-2}(0)=3.16(5) \mu \mathrm{m}^{-2}$, this gap structure accumulated in the $\lambda_{\mathrm{ac}}^{-2}(T)$ plotting completely missed describing the experimental curve of $\lambda_{\mathrm{ac}}^{-2}(T)$. Figure 5(a) shows the plotting of the $h$-wave model in the case of $a=1$, $\Delta_{\mathrm{h}}(0)=0.89 \mathrm{meV}$, and $\lambda_{\mathrm{ac}}^{-2}(0)=3.16 \mu \mathrm{m}^{-2}$. In another way, Fig. 5(b) shows the fitting result of the $h$-wave model by fixing the $a=1$ and letting parameters $\Delta_{\mathrm{h}}(0)$ and $\lambda_{\mathrm{ac}}^{-2}(0)$ be free. The fitting worked almost the same as that of the $d$-wave model obtaining the fitting parameter $\lambda_{\mathrm{ac}}^{-2}(0)=3.40(5) \mu \mathrm{m}^{-2}$ and $\Delta_{\mathrm{h}}(0)=1.36(5) \mathrm{meV}$, which overestimated the absolute value of $\lambda_{\mathrm{ac}}^{-2}(0)$. The unsuccessful gap structure is visualized in the inset where the broken lines ( $h$-wave) are overlapping the solid line ( $d$-wave). These show the beauty of the $h$-wave SC gap function that can describe both the nodal line and the flat gap structure at the same time by tuning $a$. This is also the invention to test the robustness of either $s$ - or $d$-wave model for describing a $\lambda_{\mathrm{ac}}^{-2}(T)$ curve. Besides, this trial uses the single component function to analyze the intermediate Cooper pairing gap symmetry on single FS, and to introduce the hyperbolic term to the SC phase within the application of $\mu^{+}$SR for SC materials.

To confirm our concluded steeper nodal gap compared to that of traditional $d$-wave symmetry we repeated the same analysis procedure by using $\mathrm{g}(\phi)=|\cos (2 \phi)|^{n}$, which has the periodicity from $\phi=0$ to $\pi / 4$. This function could produce the narrower nodal gap, although the flat and nodal part could not be reproduced simultaneously even tuning $n$ and had a worse $\chi^{2}$ than the best-fit $h$-wave. We found the $n=0.33(10)$ confirming the steep SC nodal line in $\lambda$-Ga. Therefore, we succeed in revealing that the nodal line with a narrower width than that of the $d$-wave and the flat part with the maximum gap exists, i.e., we reveal the most reliable $h$ symmetry to describe the superconductivity in $\lambda$-Ga.

\section{DISCUSSION}

Table I summarizes the fitting results using different gap functions. All the resulting fitting parameters summarized there were statistically significant with the $P$ value $<5 \%$ providing very strong evidence [49]. The distorted nodal line $h$-wave gap function gives the best reduced $\chi^{2}$ among tested ones yielding $\rho_{\mathrm{s}} c^{-2}=3.16(5) \mu \mathrm{m}^{-2}, \lambda_{\mathrm{ac}}(0)=560(5) \mathrm{nm}$, 
TABLE I. The fitting result of $\lambda_{\mathrm{ac}}^{-2}(T)$ in $\lambda$-(BETS) ${ }_{2} \mathrm{GaCl}_{4}$ using the different models shown in Fig. 3 . The SC gap in meV, energy ratio in $k_{\mathrm{B}} T_{c}$, goodness of the fitting, reduced $\chi^{2}$, are summarized for each tested gap function. In the $s+d$-wave symmetry, $\omega_{\mathrm{s}}$ is the weighting factor of the $s$-wave. In the $h$-wave distorted nodal line symmetry, $a$ is the factor to describe the steepness of the nodal line compared with that of the traditional $d$-wave. The $\Delta_{\mathrm{d}}, \Delta_{\mathrm{s}}, \Delta_{\mathrm{h}}$ are SC gaps obtained from the fitting by using the $s$-, $d$-, and $h$-wave symmetry, respectively [51].

\begin{tabular}{|c|c|c|c|c|c|c|}
\hline Model & $g(\phi)$ & Gap (meV) & Factors & $2 \Delta /\left(k_{\mathrm{B}} T_{c}\right)$ & $\lambda_{\mathrm{ac}}^{-2}(0)\left(\mu \mathrm{m}^{-2}\right)$ & Reduced $\chi^{2}$ \\
\hline$d$-wave & $|\cos (2 \phi)|$ & $\Delta_{\mathrm{d}}(0)=1.3(2)$ & & $5.6(9)$ & $3.41(6)$ & 5.62 \\
\hline$s$-wave & 1 & $\Delta_{\mathrm{s}}(0)=0.82$ & & $3.6(1)$ & $3.05(3)$ & 1.63 \\
\hline$s+d$-wave & $\omega_{\mathrm{s}}+\left(1-\omega_{\mathrm{s}}\right)|\cos (2 \phi)|$ & $\begin{array}{c}\Delta_{s}(0)=0.80(8) \\
\Delta_{d}(0)=1.3(5)\end{array}$ & $\omega_{s}(0)=0.71(1)$ & $\begin{array}{l}3.5(3) \\
5.6(9)\end{array}$ & $3.15(6)$ & 1.19 \\
\hline$h$-wave & $\left|\tanh \left(a\left(\phi-\phi_{0}\right)\right)\right|$ & $\Delta_{\mathrm{h}}(0)=0.89(4)$ & $a=4.6(2.1)$ & $3.9(2)$ & $3.16(5)$ & 1.08 \\
\hline
\end{tabular}

$a=4.6(2.1)$, and $2 \Delta_{\mathrm{h}} / k_{B} T_{c}=3.9(2)$. The obtained value of $\lambda_{\text {ac }}(0)$ through the $h$-wave model is consistent with the one obtained from the field dependence measurement $\sigma_{\mathrm{SC}}(H)$. From $\lambda_{\mathrm{ac}}(0)$ we obtained SC carrier density, $n_{s}=\lambda_{\mathrm{ac}} m^{*} /\left(e^{2} \mu_{0}\right)$. Using $m^{*}=3.6 m_{e}$ and $6.3 m_{e}$ [24], we obtained $n_{s}=3.22 \times$ $10^{20} \mathrm{~cm}^{-3}$ and $5.63 \times 10^{20} \mathrm{~cm}^{-3}$, respectively. These are about $57.5 \%$ and $100 \%$ of the carrier density expected assum-
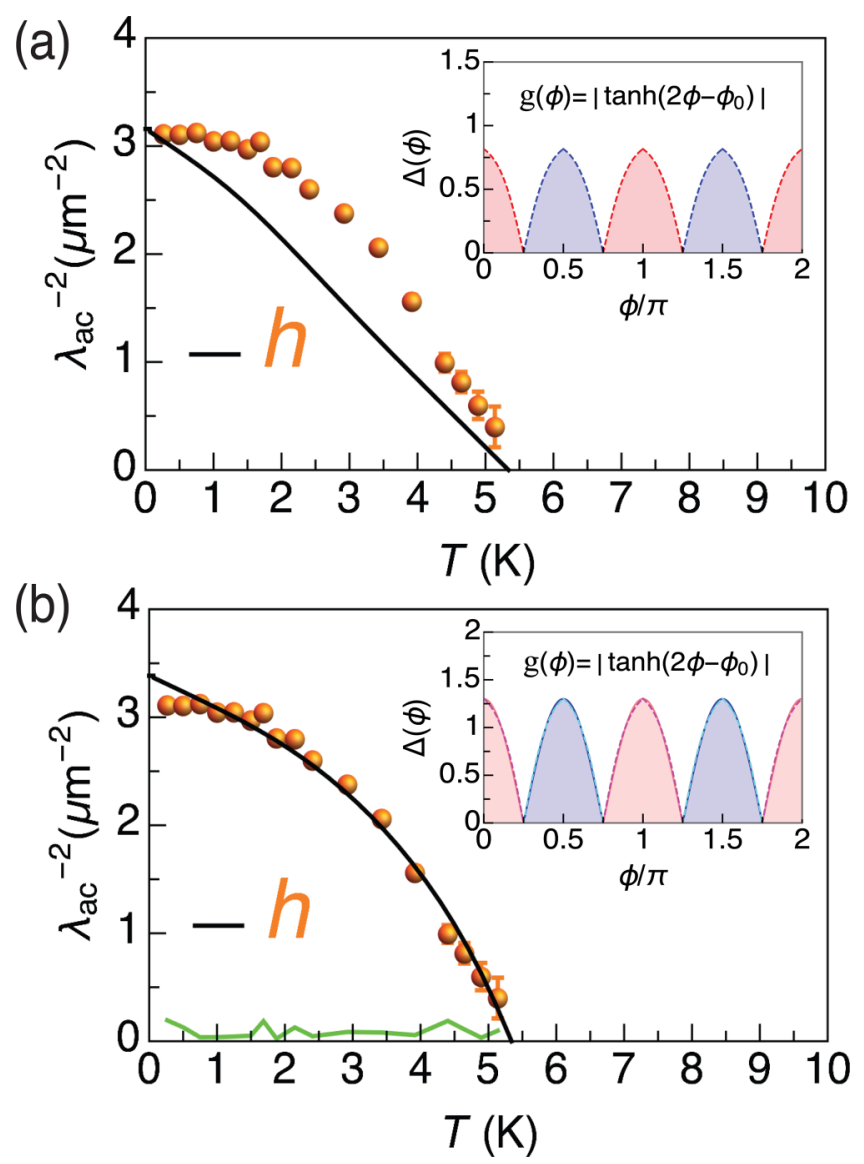

FIG. 5. (a) The plotting of the $h$-wave model in the case of $a=1$ and $\Delta_{\mathrm{h}}(0)=0.89 \mathrm{meV}, \lambda_{\mathrm{ac}}^{-2}(0)=3.16 \mu \mathrm{m}^{-2}$, the same parameters obtained in Fig. 4(d). (b) The fitting result of the $h$-wave model in case we fixed $a=1$ and let other parameters free. The obtained parameters are simulated in the inset with broken magenta and cyan lines working very similar to the one obtained by $d$-wave symmetry plotted as solid lines filled with their red and blue area which is the inset of Fig. 4(a). The solid green line shows the difference between the fitting result of the black line and the data point. ing one carrier per formula unit, respectively. This means the superconductivity is not based exclusively on carriers forming the $\mathrm{q} 2 \mathrm{D}$ pocket FS. The unfolded FS, i.e., distorted single cylindrical FS picture, is necessary to discuss the SC state.

In the thermodynamics study on $\lambda$-Ga, the gap energy was set to be $2.15 k_{\mathrm{B}} T_{c}$ which was able to describe only the fourfold gap symmetry resulting in the $d$-wave symmetry [29]. Although the further angle-resolved-heat-capacity measurement included the notion of twofold- and fourfold-like terms, the result was insufficient to describe the detailed anisotropy of the gap function due to comparably small signals from SC components [30]. Here, the $\Delta_{\mathrm{h}}$ is corresponding to the gap energy of $h$-wave symmetry and shows the intermediate value between the traditional $d$-wave [52] and the $s$-wave energy in the weak coupling limit.

It becomes intriguing that the traditional $d$-wave symmetry in near half-filling of $\kappa$-type organic superconductor [53] and hole-doped La-based cuprates [54] can be tested using $h$-wave symmetry, since both systems have single FS but theories suggest the existence of $s$-component [55] or other nonuniform $d$-wave mechanism [56]. We expect the $h$-wave is intrinsic for the $\lambda$-type organic family.

To understand the origin of $h$ symmetry, the anisotropy of the dimer lattice in $\lambda$-Ga is the plausible mediation of the $h$-wave Cooper pairing. We see it as an alternation of two sublattices in the $a$ direction which are anisotropic triangular with $t^{\prime} / t=0.8$ and frustrated squared with $t^{\prime} / t=0.2$, shown in Fig. 1(a). The two different spin moments [10] fluctuating in the metallic state recall the development of two kinds of magnetic fluctuations evidenced by ${ }^{13} \mathrm{C}-\mathrm{NMR}$ [32] whereas the small spin moment is reminiscent of a charge disproportionation evidenced by ${ }^{77} \mathrm{Se}-\mathrm{NMR}$ measurements [17,57]. The resonance valence bond theory of the Hubbard-Heisenberg model on a 1/2-filled anisotropic triangular lattice revealed that at $t^{\prime} \sim t$ spin fluctuations mediate the fully-gapped $d+$ $i d$-wave SC state [58]. Nevertheless, a variational Monte Carlo study of the 1/2-filled-band Hubbard model showed the strength of $t^{\prime} / t=0.2$ of a frustrated squared lattice stabilizes the $d$-wave SC state with nodal line in the diagonal direction, adjacent to Mott insulator and AFLRO states [59]. However, the Cooper pairing symmetry of $\lambda-\mathrm{Ga}$ is not a simple summation of the $d+i d$ - and $d$-wave since we reveal that the nodal line is steeper than that of traditional $d$-wave by using $h$-wave SC gap function. The Cooper pairs mediated by each type of spin fluctuation arrayed in the triangular and squared sublattices on the conducting 2D plane seem entangled through higher spatial dimension [60]. If this is 
the case the entanglement of two Cooper pairs is a minimum requirement, unlike the entanglement of electrons forming a Cooper pair mediated by one type of spin fluctuation arrayed in one type of lattice. In analogy with studies in the Fermi gas $[25,26]$, anisotropic exchange interaction (or anisotropic spin fluctuations $[58,61]$ ) in the $\lambda$-type crystal might then be related to the anisotropic Fermi liquid character [62], and is the first key example for organic superconductors to modify SC gap structure by distorting FS unwrapping new features of the SC nodal state beyond the traditional $d$-wave symmetry.

\section{CONCLUSION}

We have measured the temperature dependence of the inplane London penetration depth, $\lambda_{\mathrm{ac}}^{-2}(T)$, in $\lambda$-Ga by $\mu^{+} \mathrm{SR}$ and found the SC gap structure exhibits a distorted nodal line structure which contains four nodes on single FS with a narrower width than that of the traditional $d$-wave by the steepness factor of 4.6(2.1) on average alternating with a flat gap with amplitude of $2 \Delta_{\mathrm{h}} / k_{\mathrm{B}} T_{c}=3.9(2)$. The decomposition of the obtained SC gap structure into each component along the crystal axes may lead to more detailed information of this unusual Cooper pair formation, likely the same as that surviving under high magnetic field, mediated by anisotropic spin fluctuations. It should be possible by complementary measurements obtaining the temperature dependence of outof-plane penetration depth and anisotropy of Fermi liquid in the metallic state using oriented crystals in the next stage.

\section{ACKNOWLEDGMENTS}

We acknowledge H. Aizawa, T. Koretsune, H. Seo, K. Kuroki, Y. Nakazawa, M. Hagiwara, S. Nakosai, M. Ogata, and M. Iwasaki for helpful discussion and A. Berlie and F. L. Pratt for $\mu^{+}$SR experimental support. This work is supported by the Junior Research Associate Fellowship RIKEN Program, LPDP Scholarship of Indonesia, and partly supported by JSPS/MEXT Grants-in-Aids for Scientific Research (KAKENHI) Grant No. JP18K03526.
[1] Y. Kitaoka, K. Ueda, T. Kohara, K. Asayama, Y. Onuki, and T. Komatsubara, J. Magn. Magn. Mater 52, 341 (1985).

[2] Z.-X. Shen, D. S. Dessau, B. O. Wells, D. M. King, W. E. Spicer, A. J. Arko, D. Marshall, L. W. Lombardo, A. Kapitulnik, P. Dickinson, S. Doniach, J. DiCarlo, T. Loeser, and C. H. Park, Phys. Rev. Lett. 70, 1553 (1993).

[3] J. R. Kirtley, Tsuei, A. C. C., C. J. M. Verwijs, S. Harkema, and H. Hilgenkamp, Nat. Phys. 2, 190 (2006).

[4] M. C. Boyer, W. D. Wise, K. Chatterjee, M. Yi, T. Kondo, T. Takeuchi, H. Ikuta, and E. W. Hudson, Nat. Phys. 3, 802 (2007).

[5] R. Khasanov, A. Shengelaya, A. Maisuradze, F. L. Mattina, A. Bussmann-Holder, H. Keller, and K. A. Müller, Phys. Rev. Lett. 98, 057007 (2007).

[6] B. Keimer, S. A. Kivelson, M. R. Norman, S. Uchida, and J. Zaanen, Nature (London) 518, 179 (2015).

[7] T. Hanaguri, S. Kasahara, J. Böker, I. Eremin, T. Shibauchi, and Y. Matsuda, Phys. Rev. Lett. 122, 077001 (2019).

[8] L. P. Le, G. M. Luke, B. J. Sternlieb, W. D. Wu, Y. J. Uemura, J. H. Brewer, T. M. Riseman, C. E. Stronach, G. Saito, H. Yamochi, H. H. Wang, A. M. Kini, K. D. Carlson, and J. M. Williams, Phys. Rev. Lett. 68, 1923 (1992).

[9] D. Guterding, S. Diehl, M. Altmeyer, T. Methfessel, U. Tutsch, H. Schubert, M. Lang, J. Müller, M. Huth, H. O. Jeschke, R. Valentí, M. Jourdan, and H.-J. Elmers, Phys. Rev. Lett. 116, 237001 (2016).

[10] See Supplemental Material at http://link.aps.org/supplemental/ 10.1103/PhysRevB.104.224506 for the detail density functional calculation for obtaining the FS picture and the list of transfer integral between dimer before and after including $\mu^{+}$into the system, which includes Refs. [11-14,17,2023,37,58,59,61].

[11] G. Kresse and J. Furthmüller, Phys. Rev. B 54, 11169 (1996).

[12] G. Kresse and J. Furthmüller, Comput. Mater. Sci. 6, 15 (1996).

[13] J. P. Perdew, K. Burke, and M. Ernzerhof, Phys. Rev. Lett. 77, 3865 (1996).
[14] N. Marzari and D. Vanderbilt, Phys. Rev. B 56, 12847 (1997).

[15] T. Mori, J. Phys. Soc. Jpn. 87, 044705 (2018).

[16] H. Kobayashi, H. Cui, and A. Kobayashi, Chem. Rev. 104, 5265 (2004).

[17] C. Hotta, J. Phys. Soc. Jpn. 72, 840 (2003).

[18] H. Seo, C. Hotta, and H. Fukuyama, Chem. Rev. 104, 5005 (2004).

[19] S. Uji, H. Shinagawa, T. Terashima, T. Yakabe, Y. Terai, M. Tokumoto, A. Kobayashi, H. Tanaka, and H. Kobayashi, Nature (London) 410, 908 (2001).

[20] H. Tanaka, A. Kobayashi, A. Sato, H. Akutsu, and H. Kobayashi, J. Am. Chem. Soc. 121, 760 (1999).

[21] T. Mori and M. Katsuhara, J. Phys. Soc. Jpn. 71, 826 (2002).

[22] H. Kobayashi, H. Tomita, T. Naito, A. Kobayashi, F. Sakai, T. Watanabe, and P. Cassoux, J. Am. Chem. Soc. 118, 368 (1996).

[23] H. Aizawa, T. Koretsune, K. Kuroki, and H. Seo, J. Phys. Soc. Jpn. 87, 093701 (2018).

[24] C. Mielke, J. Singleton, M.-S. Nam, N. Harrison, C. C. Agosta, B. Fravel, and L. K. Montgomery, J. Phys.: Condens. Matter 13, 8325 (2001).

[25] K. Aikawa, S. Baier, A. Frisch, M. Mark, C. Ravensbergen, and F. Ferlaino, Science 345, 1484 (2014).

[26] B. Laburthe-Tolra, Physics 5, 58 (2012).

[27] T. Suzuki, E. Negishi, H. Uozaki, H. Matsui, and N. Toyota, Physica C: Superconductivity 440, 17 (2006).

[28] S. Yasuzuka, S. Uji, T. Terashima, S. Tsuchiya, K. Sugii, B. Zhou, A. Kobayashi, and H. Kobayashi, J. Phys. Soc. Jpn. 83, 013705 (2014).

[29] S. Imajo, N. Kanda, S. Yamashita, H. Akutsu, Y. Nakazawa, H. Kumagai, T. Kobayashi, and A. Kawamoto, J. Phys. Soc. Jpn. 85, 043705 (2016).

[30] S. Imajo, S. Yamashita, H. Akutsu, H. Kumagai, T. Kobayashi, A. Kawamoto, and Y. Nakazawa, J. Phys. Soc. Jpn. 88, 023702 (2019). 
[31] T. Kobayashi, H. Taniguchi, A. Ohnuma, and A. Kawamoto, Phys. Rev. B 102, 121106(R) (2020).

[32] T. Kobayashi and A. Kawamoto, Phys. Rev. B 96, 125115 (2017).

[33] I. Watanabe, D. P. Sari, R. Asih, K. Hiraki, Y. Ishii, and T. Nakano, Superconducting Pairing Symmetry of the NonMagnetic Anion-Based Organic Superconductor $\lambda-B E T S_{2} \mathrm{GaCl}_{4}$ Studied by $\mu S R$ (STFC ISIS Neutron and Muon Source, 2020).

[34] D. P. Sari, K. Hiraki, T. Nakano, M. Hagiwara, Y. Nozue, T. Kusakawa, A. Hori, A. Yamamoto, I. Watanabe, and Y. Ishii, in Functional Properties of Modern Materials II, Materials Science Forum, Vol. 966 (Trans Tech Publications Ltd., Sanur, Bali, Indonesia, 2019), pp. 296-301.

[35] F. Pratt, S. Blundell, I. Marshall, T. Lancaster, S. Lee, A. Drew, U. Divakar, H. Matsui, and N. Toyota, Polyhedron 22, 2307 (2003), Proceedings of the 8th International Conference on Molecule-Based Magnets (ICMM 2002).

[36] F. L. Pratt and S. J. Blundell, Phys. Rev. Lett. 94, 097006 (2005).

[37] M. R. Ramadhan, I. Ramli, M. D. Umar, S. Winarsih, D. P. Sari, A. Manaf, B. Kurniawan, M. I. Mohamed-Ibrahim, S. Sulaiman, and I. Watanabe, in Functional Properties of Modern Materials II, Materials Science Forum, Vol. 966 (Trans Tech Publications Ltd., Valencia, Spain, 2019), pp. 465-470.

[38] M. Weber, A. Amato, F. N. Gygax, A. Schenck, H. Maletta, V. N. Duginov, V. G. Grebinnik, A. B. Lazarev, V. G. Olshevsky, V. Y. Pomjakushin, S. N. Shilov, V. A. Zhukov, B. F. Kirillov, A. V. Pirogov, A. N. Ponomarev, V. G. Storchak, S. Kapusta, and J. Bock, Phys. Rev. B 48, 13022 (1993).

[39] E. H. Brandt, Phys. Rev. B 37, 2349 (1988).

[40] M. Tinkham, Introduction to Superconductivity 2nd edition (Dover Publications, Inc., New York, 1996).

[41] M. Tanatar, T. Ishiguro, H. Tanaka, A. Kobayashi, and H. Kobayashi, J. Supercond. 12, 511 (1999).

[42] E. H. Brandt, Phys. Rev. B 68, 054506 (2003).

[43] F. K. K. Kirschner, F. Lang, C. V. Topping, P. J. Baker, F. L. Pratt, S. E. Wright, D. N. Woodruff, S. J. Clarke, and S. J. Blundell, Phys. Rev. B 94, 134509 (2016).

[44] M. Lang, N. Toyota, T. Sasaki, and H. Sato, Phys. Rev. Lett. 69, 1443 (1992).

[45] T. Mori, Electronic Properties of Organic Conductors (Springer, Tokyo, Japan, 2016).
[46] W. Barford and J. M. F. Gunn, Physica C Superconductivity 156, 515 (1988).

[47] A. Carrington and F. Manzano, Physica C: Superconductivity Applications 385, 205 (2003).

[48] D. P. Sari, R. Asih, S. S. Mohm-Tajudin, N. Adam, K. Hiraki, Y. Ishii, T. Takahashi, T. Nakano, Y. Nozue, S. Sulaiman, M. I. Mohamed-Ibrahim, and I. Watanabe, IOP Conf. Ser.: Mater. Sci. Eng. 196, 012047 (2017).

[49] I. G. Hughes and T. P. A. Hase, Measurements and their Uncertainties (Oxford University Press Inc., New York, 2010).

[50] A. C. Jacko, J. O. Fjærestad, and B. J. Powell, Nat. Phys. 5, 422 (2009).

[51] The SC gap equation was implemented in the language of Mathematica software for fitting the $\lambda_{a c}^{-2}(T)$ shown in Fig. 3, and all the resulting fitting parameters summarized in Table I were statistically significant with the $P$ value $\leqslant 0.0486$.

[52] K. A. Musaelian, J. Betouras, A. V. Chubukov, and R. Joynt, Phys. Rev. B 53, 3598 (1996).

[53] Y. Kawasugi, K. Seki, S. Tajima, J. Pu, T. Takenobu, S. Yunoki, H. M. Yamamoto, and R. Kato, Sci. Adv. 5, eaav7282 (2019).

[54] Z. Guguchia, D. Das, C. N. Wang, T. Adachi, N. Kitajima, M. Elender, F. Brückner, S. Ghosh, V. Grinenko, T. Shiroka, M. Müller, C. Mudry, C. Baines, M. Bartkowiak, Y. Koike, A. Amato, J. M. Tranquada, H.-H. Klauss, C. W. Hicks, and H. Luetkens, Phys. Rev. Lett. 125, 097005 (2020).

[55] H. Watanabe, H. Seo, and S. Yunoki, Nat. Commun. 10, 3167 (2019).

[56] P. Corboz, T. M. Rice, and M. Troyer, Phys. Rev. Lett. 113, 046402 (2014).

[57] K.-i. Hiraki, M. Kitahara, T. Takahashi, H. Mayaffre, M. Horvatić, C. Berthier, S. Uji, H. Tanaka, B. Zhou, A. Kobayashi, and H. Kobayashi, J. Phys. Soc. Jpn. 79, 074711 (2010).

[58] B. J. Powell and R. H. McKenzie, Phys. Rev. Lett. 98, 027005 (2007).

[59] H. Yokoyama, M. Ogata, and Y. Tanaka, J. Phys. Soc. Jpn 75, 114706 (2006).

[60] S. Sachdev, Sci. Am. 308, 44 (2013).

[61] H. Seo and H. Fukuyama, J. Phys. Soc. Jpn. 66, 3352 (1997).

[62] I. Esterlis, H. Guo, A. A. Patel, and S. Sachdev, Phys. Rev. B 103, 235129 (2021). 